\title{
Combined Detection of HER2, Ki67, and GSTP1 Genes on the Diagnosis and Prognosis of Breast Cancer
}

\author{
Bo Song, ${ }^{1, *}$ Lu Wang, ${ }^{2, *}$ Yang Zhang, ${ }^{2}$ Ning Li, ${ }^{2}$ Hao Dai, ${ }^{2}$ Huafang Xu, ${ }^{2}$ Haifeng Cai, ${ }^{2}$ and Jinyin Yan $^{2}$
}

\begin{abstract}
Objective: Breast cancer (BC) is a common malignant tumor in females. The combined assay of multiple molecular markers benefits the diagnosis and prognostic prediction. Human epidermal growth factor receptor 2 (HER2) facilitates the proliferation and differentiation of cancer cells through ligand binding. Ki67 is a tumor proliferation-related gene, whereas GSTP1 is a DNA repair-related gene. This study thus investigated the significance of HER2 and Ki67/GSTP1 gene combined assay in the diagnosis and prognosis of BC.

Materials and Methods: A total of 86 breast tumor tissues and adjacent tissues were collected. Gene expression and protein levels of HER2 and Ki67 were quantified by real-time polymerase chain reaction (PCR) and Western blot, respectively. Methylation frequency of GSTP1 was analyzed by methylation-specific PCR. The correlation between HER2 and Ki67/GSTP1 and clinical/pathological features of BC was analyzed.

Results: Gene and protein expression levels of HER2 and Ki67 in tumor tissues were increased $(p<0.05$ compared with adjacent tissues). Methylation frequency of GSTP1 gene was 37.2\%, which was significantly higher in breast tumor tissues than in adjacent tissues $(12.79 \%, p<0.05)$. HER2 expression was positively correlated with TNM stage, tumor size, and lymph node metastasis, and negatively correlated with tissue grade and estrogen receptor (ER)/progesterone receptor (PR) expression $(p<0.05)$. GSTP1 methylation was positively correlated with TNM stage and tumor size, and negatively correlated with ER/PR expression $(p<0.05)$.

Conclusions: HER2, Ki67, and GSTP1 methylation were correlated with clinical and pathological features of $\mathrm{BC}$. The combined assay benefits the early diagnosis and prognostic prediction of cancer.
\end{abstract}

Keywords: breast cancer, gene methylation, GSTP1, human epidermal growth factor receptor 2, Ki67

\section{Introduction}

B reast cancer $(\mathrm{BC})$ is the most common female-specific tumor worldwide. Its incidence is increasing by years, with the trend of younger age of patients, thus severely affecting economy and population health. ${ }^{1,2}$ The occurrence and progression of $\mathrm{BC}$ are complicated by various endogenous or exogenous factors, including environment, life style/habitat, endocrine level, and genetics. ${ }^{3,4}$ Owing to insidious onset, BC is difficult to be diagnosed in time. Majority of patients were already at terminal stage with metastasis. Therefore, early screening, diagnosis, and treatment of $\mathrm{BC}$ are critical for the improvement of patient life quality and survival time. ${ }^{5,6}$

With the advancement of molecular biology, molecular diagnostic technique has been demonstrated to benefit the individualized diagnosis and prognostic judgment of $\mathrm{BC}$, and has drawn lots of research interests. The application of molecular diagnosis such as genetic assay can benefit the diagnosis of $\mathrm{BC}$, and assist the judgment of prognosis, providing reference for the selection of treatment plan and prognostic evaluation. Therefore, molecular diagnosis has become an important component of diagnosis and treatment of BC. ${ }^{7}$ However, single factor analysis for the early diagnosis and screening of $\mathrm{BC}$ still has problems such as low sensitivity or specificity. ${ }^{8,9}$ The discovery of human epidermal growth factor receptor 2 (HER2) gene has important implications. As a member of epidermal growth factor receptor (EGFR) family, HER2 gene is an oncogene. Previous studies showed the close correlation of HER2 gene or protein expression abnormality with the occurrence, progression,

\footnotetext{
${ }^{1}$ Breast Surgery Department, Shandong Tengzhou Maternity and Children Care Hospital, Tengzhou, China.

${ }^{2}$ Department of Surgical Oncology, Tangshan People's Hospital, Tangshan, Hebei, China.

*These authors contributed equally to this work.
}

Address correspondence to: Jinyin Yan; Department of Surgical Oncology, Tangshan People's Hospital; No. 65 of Shengli Road, Lunan District, Tangshan 063000, Hebei, China

E-mail: cisoa2kch4rv@sina.com 
invasion, and metastasis of $\mathrm{BC} .^{10,11} \mathrm{Ki}-67$ is a nuclear protein that is related with ribosomal RNA transcription, and belongs to tumor proliferation-related gene ${ }^{12}$ to reflect cell proliferation status. Ki-67 is continuously expressed in all phases (G1, S, G2, and $\mathrm{M}$ ) of cell proliferation except G0 phase. ${ }^{13}$ The abnormal expression of Ki-67 is closely related with differentiation and tumor metastasis of $\mathrm{BC} .^{13,14}$ GSTP1 is a DNA repair-related gene and its methylation frequency is directly correlated with tumor occurrence and progression. ${ }^{15}$ Considering the role of HER2, Ki67, and GSTP1 in the pathogenesis of BC, whether combined measurement of these parameters is beneficial for the diagnosis and prognosis of $\mathrm{BC}$ remains poorly understood. This study aimed to investigate the combined gene assay of HER2, Ki67, and GSTP1 in the diagnosis and prognostic prediction of $\mathrm{BC}$.

\section{Materials and Methods}

\section{General information}

A total of 86 BC patients who were diagnosed by pathohistological examination from January 2015 to December 2015 were recruited in Tangshan People's Hospital. All included patients received surgery. The age of patients ranges from 38 to 62 years, with an average age $=45.1 \pm 6.6$ years.

Inclusion criteria. The criteria for inclusion were confirmed diagnosis of primary BC by pathology examination, primary tumor onset, not receiving surgery, chemotherapy, or radiotherapy.

Exclusion criteria. The criteria for exclusion were recurrent BC, history of tumor treatment, complication with other diseases such as infectious disease, malignant tumor, severe liver/kidney disorder, pulmonary fibrosis, bone metabolic disorder, secondary renal hypertension, systemic immune disease, and complication of malignant tumors, or those patients who did not comply or were unable to comply with this study and its follow-up. Clinical and pathological features of $\mathrm{BC}$ patients are given in Table 1. Tumor and adjacent tissue samples were collected during surgery for pathology diagnosis and typing/grading of tumors, which were all diagnosed as primary $\mathrm{BC}$ by pathology examination. Both tumor and adjacent tissues were collected and frozen at $-80^{\circ} \mathrm{C}$. This study was approved by the ethical committee of Tangshan People's Hospital. All participants have signed written consent forms.

\section{Treatment of $B C$}

All included BC patients received combined assay using surgery as the major step. Through assisted diagnosis of preoperative routine examination and imaging, and biopsy of frozen tissues during the surgery, all patients were diagnosed as having primary BC. Improved radical surgery of $\mathrm{BC}$ was performed in conjunction with clearance of pit lymph node based on the metastatic condition of patients. Tissue samples were collected during surgery for the judgment of clinical stage and metastatic condition.
Table 1. Clinical Parameters and Pathological Features of Breast Cancer Patients

\begin{tabular}{|c|c|c|}
\hline & Case No. (n) & $\%$ \\
\hline \multicolumn{3}{|l|}{ Age (year) } \\
\hline$\leq 40$ & 38 & 44.19 \\
\hline$>40$ & 48 & 55.81 \\
\hline \multicolumn{3}{|l|}{ BMI $\left(\mathrm{kg} / \mathrm{m}^{2}\right)$} \\
\hline$\leq 23$ & 42 & 48.83 \\
\hline$>23$ & 44 & 51.17 \\
\hline \multicolumn{3}{|l|}{ Family history of BC } \\
\hline Yes & 36 & 41.86 \\
\hline No & 50 & 58.14 \\
\hline \multicolumn{3}{|l|}{ Tumor size } \\
\hline $\mathrm{T} 1(<2 \mathrm{~cm})$ & 23 & 26.74 \\
\hline $\mathrm{T} 2(2-5 \mathrm{~cm})$ & 17 & 19.76 \\
\hline $\mathrm{T} 3(>5 \mathrm{~cm})$ & 18 & 20.93 \\
\hline T4 (tumor invasion) & 28 & 32.57 \\
\hline \multicolumn{3}{|l|}{ Histology grade } \\
\hline High differentiation & 25 & 29.07 \\
\hline Moderate differentiation & 33 & 38.37 \\
\hline Low differentiation & 28 & 32.56 \\
\hline \multicolumn{3}{|l|}{ TNM stage } \\
\hline I-II & 32 & 37.21 \\
\hline III-IV & 54 & 62.79 \\
\hline \multicolumn{3}{|l|}{ Lymph node metastasis } \\
\hline No & 34 & 39.53 \\
\hline Yes & 52 & 60.47 \\
\hline \multicolumn{3}{|l|}{ PR } \\
\hline Positive & 35 & 40.69 \\
\hline Negative & 51 & 59.31 \\
\hline \multicolumn{3}{|l|}{$\mathrm{ER}$} \\
\hline Positive & 30 & 34.88 \\
\hline Negative & 56 & 65.12 \\
\hline
\end{tabular}

$\mathrm{BC}$, breast cancer; ER, estrogen receptor; $\mathrm{PR}$, progesterone receptor.

\section{Record of clinical information}

Both clinical information and patient record were collected for measuring estrogen receptor (ER) and progesterone receptor $(\mathrm{PR})$ expression profiles for patients. Tumor size, pathology grade, and clinical stage were used to analyze the condition of tumor metastasis.

\section{HER2 and Ki67 gene expression in tumor and adjacent tissues by real-time polymerase chain reaction}

Trizol reagent was used to extract total RNA from tumor tissue and adjacent tissues. DNA reverse transcription was performed following the instruction of the kit (Axygen). Primers were designed by Primer6.0 software and synthesized by Invitrogen (China). Primer sequences were HER2forward, 5'-CATGG TATGA TGGAC C-3'; HER2-reverse, 5'-TTCGA AGTCG CACAG CAGCT-3'; Ki67-forward, 5' CATGG TATGA TGATG GGTAG ACC-3'; Ki67-reverse, 5'TTCAC AAGGA CAAGT CGCAG CAGCT-3'; GAPDHforward, 5'-ACCAG GTATC TGCTG GTT-3'; GAPDHreverse, and 5'-TAACC ATGAT GTCAG CGTG GTT-3'. Real-time polymerase chain reaction (PCR) was performed to measure the target gene expression using the following conditions: $55^{\circ} \mathrm{C}$ for $1 \mathrm{~min}$, followed by 35 cycles each containing $92^{\circ} \mathrm{C}$ for $30 \mathrm{~s}, 58^{\circ} \mathrm{C}$ for $45 \mathrm{~s}$, and $72^{\circ} \mathrm{C}$ for $35 \mathrm{~s}$. 


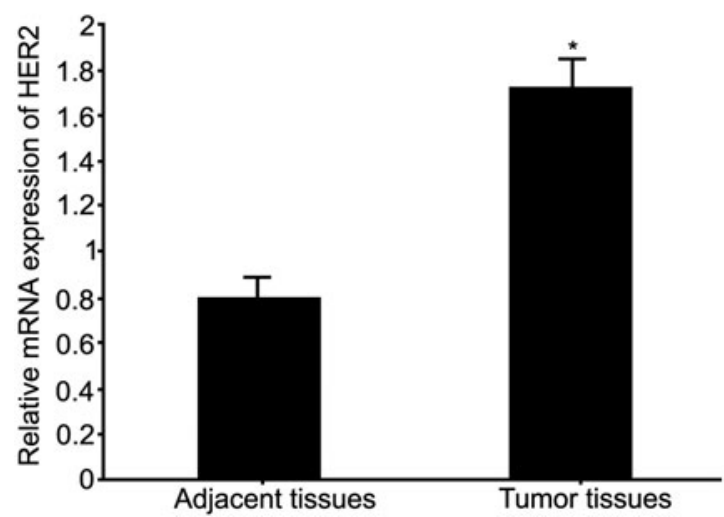

FIG. 1. HER2 gene expression level in BC tissues. $* p<0.05$ compared with adjacent tissues. BC, breast cancer; HER2, human epidermal growth factor receptor 2 .

Data were collected by built-in software in the cycler. Using GAPDH as the reference, CT values of all samples and standards were calculated based on fluorescent quantification. Using CT values of standards as the reference, standard curve was plotted, followed by semiquantitative analysis using the $2^{-\Delta \mathrm{Ct}}$ method.

\section{Western blot for detecting HER2 and Ki67 protein expression}

Proteins were extracted from tumor and adjacent tissues by routine procedures and stored at $-20^{\circ} \mathrm{C}$ for Western blotting analysis. In brief, proteins were separated by $10 \%$ SDS-PAGE and transferred to polyvinylidene fluoride membrane by semidry method in $100 \mathrm{~V}$ constant electrical field for $1.5 \mathrm{~h}$. Nonspecific background was removed through incubation with $5 \%$ defatted mild powders for $2 \mathrm{~h}$. AntiHER2 or Ki67 monoclonal antibody (1:1000 and 1:2000 dilution) was added and incubated overnight at $4^{\circ} \mathrm{C}$. After rinsing in phosphate buffered saline with Tween-20 (PBST) at room temperature, human resource planningconjugated goat antirabbit secondary antibody (1:2000) was added for $30 \mathrm{~min}$ incubation, followed by PBST rinsing and enhanced chemiluminescence (ECL) development for $1 \mathrm{~min}$. With X-ray exposure, results were observed and scanned using protein imaging system and Quantity One software for measuring band density. Each experiment was repeated four times $(n=4)$ for statistical analysis.

\section{Immunohistochemistry staining}

BC tissues and adjacent tissues were fixed in $4 \%$ paraformaldehyde and embedded for section with a thickness of $3 \mu \mathrm{m}$. After dewaxing, the slice was blocked by $0.03 \% \mathrm{H}_{2} \mathrm{O}_{2}$ to inhibit the endogenous activity of $\mathrm{H}_{2} \mathrm{O}_{2}$. After that, the slice was incubated with citrate buffer $(\mathrm{pH}=7.4)$ for antigen retrieval followed by addition of $3 \%$ FBS and subsequent incubation for 20 min to block the nonspecific binding. Then the slice was incubated with primary antibody against HER2 (dilution 1:1000) or Ki67 (1:1500) at $4^{\circ} \mathrm{C}$ overnight followed by incubation with secondary antibody at room temperature for $60 \mathrm{~min}$. The slice was detected after development by $\mathrm{DAB}-\mathrm{H}_{2} \mathrm{O}_{2}$ for $20 \mathrm{~min}$.

\section{Methylation-specific PCR for measuring GSTP1 methylation}

DNeasy tissue kit and EZ DNA Methylation-Gold kit (QIAGEN) were used to extract and purify genomic DNA from tumor and adjacent tissues following manual instructions. GSTP1 gene methylation primers were designed as follows: forward, 5'-TTCGG GGTGT AGCGG TCGTC-3'; reverse, 5'-GCCCC AATAC TAAAT CACGA CG-3. GSTP1 nonmethylation primers were forward, 5'-GATGT TTGGG GTGTA GTGGT TGTT-3'; reverse, 5'-CCACC CCAAT ACTAA ATCAC AACA-3'. PCR was conducted in a $15 \mu \mathrm{L}$ reaction system consisting of DNA template, $1.5 \mu \mathrm{L}$ sulfite, $0.45 \mu \mathrm{L}$ dNTPs $(20 \mathrm{mmol} / \mathrm{L}), 0.15 \mu \mathrm{L}$ of both
A
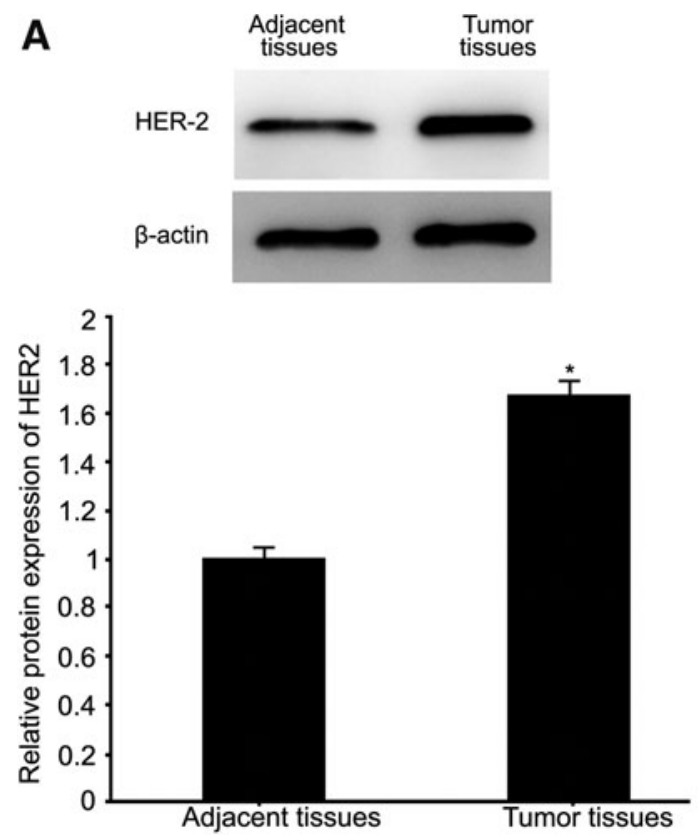

B

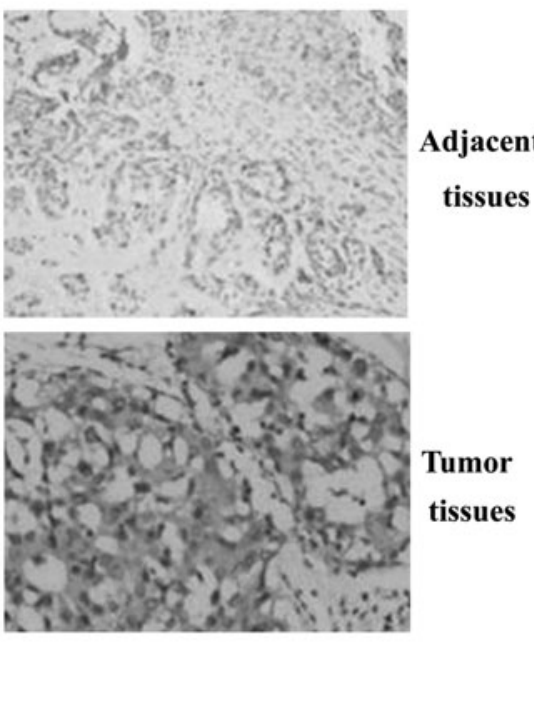

FIG. 2. HER2 protein expression level in $\mathrm{BC}$ tissues as measured by Western blot (A) and immunohistochemistry staining (B) $(40 \times) .{ }^{*} p<0.05$ compared with adjacent tissues. 


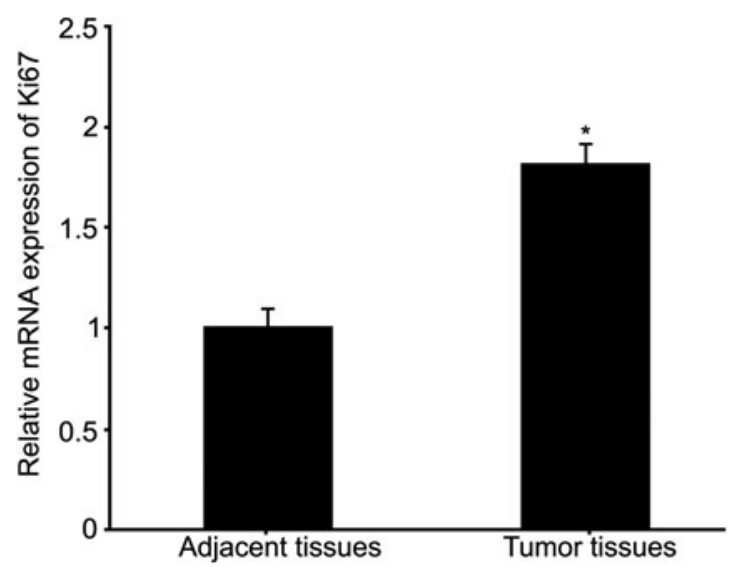

FIG. 3. Ki67 gene expression level in BC tissues. $* p<0.05$ compared with adjacent tissues.

forward and reverse primers $(20 \mathrm{nmol} / \mathrm{L}), 0.12 \mu \mathrm{L}$ Hotstar Taq DNA polymerase, and distilled water.

\section{Statistical analysis}

All data were analyzed by SPSS22.0 software. Measurement data are presented as mean \pm standard deviation. Oneway analysis of variance (ANOVA) was used to compare means among multiple groups. Least significant difference test was used to compare between two groups. Pearson analysis was used to assess the correlation. A statistical significance was identified when $p<0.05$.

\section{Results}

\section{HER2 gene expression level of BC tissues}

Real-time PCR was used to test HER2 gene expression level in tumor tissues of BC patients. Results showed sig- nificantly elevated expression of HER2 gene in BC tissues $(p<0.05$ compared with adjacent tissues, Fig. 1).

\section{HER2 protein expression in $B C$ tissues}

Western blotting was used to quantify HER2 protein expression level in BCs. Results showed that, similar to HER2 gene expression level, HER2 protein was significantly upregulated in $\mathrm{BC}$ tissues $(p<0.05$ compared with adjacent tissues, Fig. 2A). Consistently, immunohistochemistry staining showed increased expression of HER2 protein in $\mathrm{BC}$ tissues compared with adjacent tissues (Fig. 2B).

\section{Ki67 gene expression in $B C$ tissues}

Real-time PCR was used to quantify Ki67 gene expression level in BC tissues. Results showed significantly elevated Ki67 gene expression in BC tissues $(p<0.05$ compared with adjacent tissues, Fig. 3).

\section{Ki67 protein expression in BC tissues}

Western blotting was used to test Ki67 protein expression level in BC tissues. Results showed significantly elevated Ki67 protein expression in tumor tissues $(p<0.05$ compared with adjacent tissues, Fig. 4A). In accordance with the expression profile of $\mathrm{Ki} 67$ measured by Western blot, immunohistochemistry staining showed elevated expression of $\mathrm{Ki} 67$ protein in $\mathrm{BC}$ tissues compared with adjacent tissues (Fig. 4B).

\section{GSTP1 methylation level in BC tissues}

Methylation-specific PCR was used to test the methylation level of GSTP1 gene in BC tissues. Results showed the frequency of GSTP1 methylation at 37.2\% (32/86), which was significantly higher in BC tissues than in adjacent tissues $(12.79 \%, 11 / 86, p<0.05$, Table 2$)$.
FIG. 4. Ki67 protein expression levels in $\mathrm{BC}$ tissues as measured by Western blot (A) and immunohistochemistry staining $($ B) $(40 \times)$. $* p<0.05$ compared with adjacent tissues.

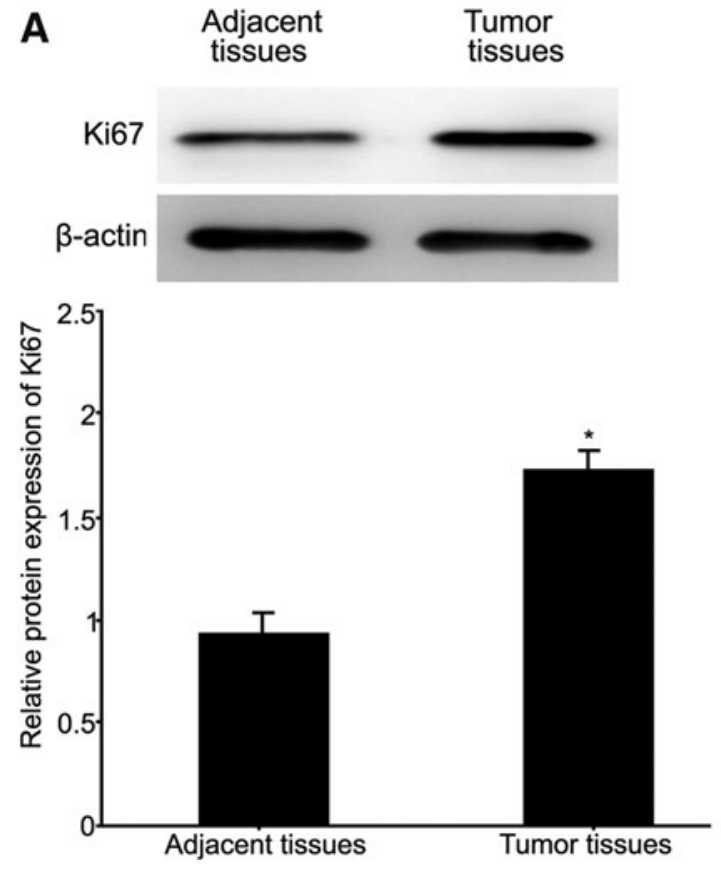

B

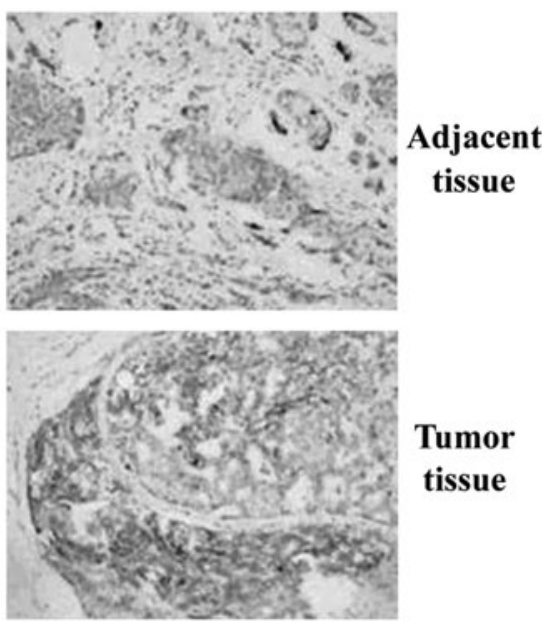


Table 2. GSTP1 Methylation Level in Breast Cancer Tissues

\begin{tabular}{lccccc}
\hline & \multicolumn{2}{c}{ Methylation } & & \multicolumn{2}{c}{ Nonmethylation } \\
\cline { 2 - 3 } \cline { 6 - 7 } GSTP1 & $\mathrm{n}$ & $\%$ & & $\mathrm{n}$ & $\%$ \\
\hline Adjacent tissue & 11 & 12.79 & & 75 & 87.21 \\
BC tissue & 32 & 37.20 & & 54 & 62.80 \\
\hline
\end{tabular}

\section{Correlation between HER2, Ki67, GSTP1 methylation and clinical/pathological features}

HER2 expression was positively correlated with TNM stage, tumor size, and lymph node metastasis, and negatively correlated with tissue grade or ER/PR expression $(p<0.05)$. Ki67 expression was positively correlated with TNM stage and lymph node metastasis, and negatively correlated with tissue grade and ER/PR expression $(p<$ 0.05). GSTP1 methylation was positively correlated with TNM stage and tumor size, and negatively correlated with ER/PR expression $(p<0.05$, Table 3$)$.

\section{Discussion}

The occurrence and development of BC involve abnormal activation of oncogenes and inactivation of tumor inhibitor genes. ${ }^{16}$ Owing to insidious onset, early $\mathrm{BC}$ does not have typical symptoms and does not attract attention. Patients are often misdiagnosed or receive late diagnosis. They frequently received confirmed diagnosis until the development of significant clinical symptoms. However, some patients often had malignant transformation or metastasis, causing challenges in treatment. ${ }^{17}$ The early diagnosis and precise evaluation of prognosis significantly improve patient quality of life. Molecular diagnosis has become a novel technique for $\mathrm{BC}$ diagnosis. However, different parameters of genetic assays have unique implications, along with various correlations with clinical or pathological features of BC. Therefore, the combination of different genetic assays benefits diagnosis and prognostic prediction of BC. ${ }^{18}$

HER2 gene encodes proteins, which are specifically coupled with ligands to form heterodimers, which cause conformational change of HER2 proteins, and autophosphorylation of tyrosine kinase inside the cytoplasm to activate signal pathways. The activity of this heterodimer is advantageous over heterodimers that are formed by other members in EGFR family, causing a cascade reaction. ${ }^{19,20}$ HER2 protein and ligand participate in physiological activities of cells to facilitate their activation, proliferation, and differentiation. ${ }^{21}$ The occurrence, development, and invasion or metastasis of tumors is correlated with HER2 overexpression. $^{22}$ Consistent with these previous studies, in this study, it has been shown that HER2 expression was positively correlated with clinical TNM stage, tumor size, and lymph node metastasis, and negatively correlated with ER/PR expression and tissue grade, suggesting that HER2 expression might be served as a molecular marker for diagnosis and prognostic analysis of BC.

The precise biological function of Ki67 has not been fully illustrated. As Ki67 is closely related with cell proliferation, most studies have agreed on its value in evaluating cell proliferation grade. ${ }^{23}$ This study confirmed significantly elevated expression of Ki67 gene and protein expression, both of which were positively correlated with TNM stage and lymph node metastasis, and negatively correlated with tissue grade and ER/PR expression. The change of functional genes regulating cell cycle or DNA repair is closely related with $\mathrm{BC}$ occurrence. The methylation of DNA repair gene causes its functional inactivation, further leading to tumor occurrence. Therefore, as a novel marker in molecular biology, gene methylation study can contribute to the analysis of pathogenesis and treatment plan of BC. As a member of metabolic enzyme family, GSTP1 is a DNA repair-related gene, and protects DNA from damage, regulates metabolism and detoxification, thus inhibiting tumor occurrence. The methylation of GSTP1 gene often indicates progression of tumors, including BC or unfavorable prognosis. ${ }^{24,25}$ Consistent with the role of GSTP1 methylation in the progression of tumors, this study demonstrated significantly elevated methylation frequency of GSTP1 gene in BC tissues, which was positively correlated with TNM stage and tumor size, and negatively correlated with ER/PR expression. These results indicate the beneficial effects of combined assay of three molecular markers in the diagnosis and prognostic prediction of $\mathrm{BC}$. However, owing to limited number of patients enrolled in this study, which is a main study limitation, large cohort clinical studies are required to confirm these findings in the future.

\section{Conclusions}

The elevation of HER2/Ki67 expression and GSTP1 gene methylation in BC tissues is closely correlated with clinical and pathological features of $\mathrm{BC}$, indicating that these genes might be molecular markers for tumor diagnosis and prognostic prediction. The combined assay of these genes might benefit the early diagnosis and prognostic evaluation of $\mathrm{BC}$, thus providing evidence for clinical treatment of BC.

Table 3. Correlation Between Human Epidermal Growth Factor Receptor 2, Ki67, or GSTP1 Methylation and Clinical/Pathological Features

\begin{tabular}{|c|c|c|c|c|c|}
\hline & $\begin{array}{l}\text { TNM } \\
\text { stage }\end{array}$ & $\begin{array}{l}\text { Tumor } \\
\text { size }\end{array}$ & $\begin{array}{l}\text { Lymph node } \\
\text { metastasis }\end{array}$ & $\begin{array}{c}E R / P R \\
\text { expression }\end{array}$ & $\begin{array}{l}\text { Tissue } \\
\text { grade }\end{array}$ \\
\hline HER2 & $0.635^{*}$ & $0.727 *$ & $0.815^{*}$ & $-0.521 *$ & $-0.615^{*}$ \\
\hline Ki67 & $0.689 *$ & 0.142 & $0.652 *$ & $-0.663^{*}$ & $-0.525^{*}$ \\
\hline $\begin{array}{l}\text { GSTP1 } \\
\text { methylation }\end{array}$ & $0.711 *$ & $0.528 *$ & 0.312 & $-0.678 *$ & -0.162 \\
\hline
\end{tabular}

$* p<0.05$.

HER2, human epidermal growth factor receptor 2. 


\section{Acknowledgment}

This work was supported by the Tangshan science and technology project (No. 13130275b).

\section{Disclosure Statement}

No competing financial interests exist.

\section{References}

1. Li R, Hu S, Chang Y, et al. Development and characterization of a humanized anti-HER2 antibody HuA21 with potent anti-tumor properties in breast cancer cells. Int J Mol Sci $2016 ; 17$.

2. Thavendiranathan P, Abdel-Qadir H, Fischer HD, et al. Breast cancer therapy-related cardiac dysfunction in adult women treated in routine clinical practice: A populationbased cohort study. J Clin Oncol 2016;34:2239-2246.

3. Tu DG, Yu Y, Lee CH, et al. Hinokitiol inhibits vasculogenic mimicry activity of breast cancer stem/progenitor cells through proteasome-mediated degradation of epidermal growth factor receptor. Oncol Lett 2016;11: 2934.

4. Jacob L, Hadji P, Kostev K. Age-related differences in persistence in women with breast cancer treated with tamoxifen or aromatase inhibitors in Germany. J Geriatr Oncol 2016;7:169-175.

5. Lai Y, Chen Y, Wu S, et al. Modified volumetric modulated arc therapy in left sided breast cancer after radical mastectomy with flattening filter free versus flattened beams. Medicine (Baltimore) 2016;95:e3295.

6. Li J, Wang J, Li M, et al. Up-regulated expression of scavenger receptor class B type 1 (SR-B1) is associated with malignant behaviors and poor prognosis of breast cancer. Pathol Res Pract 2016;212:555-559.

7. O'Neill SC, Isaacs C, Chao C, et al. Adoption of gene expression profiling for breast cancer in US oncology practice for women younger than 65 years. J Natl Compr Canc Netw 2015;13:1216.

8. De Silva N, Schulz L, Paterson A, et al. Molecular effects of Lapatinib in the treatment of HER2 overexpressing oesophago-gastric adenocarcinoma. Br J Cancer 2015;113: 1305.

9. He J, Shi H, Zhou Z, et al. Correlation between apparent diffusion coefficients and HER2 status in gastric cancers: Pilot study. BMC Cancer 2015;15:749.

10. Hongisto V, Aure MR, Mäkelä R, et al. The HER2 amplicon includes several genes required for the growth and survival of HER2 positive breast cancer cells-A data description. Genom Data 2014;2:249.

11. Shilova ON, Proshkina GM, Lebedenko EN, et al. Internalization and recycling of the HER2 receptor on human breast adenocarcinoma cells treated with targeted phototoxic protein DARPinminiSOG. Acta Naturae 2015;7:126.
12. Maselli A, Capoccia S, Pugliese P, et al. Autoantibodies specific to estrogen receptor alpha act as estrogen agonists and their levels correlate with breast cancer cell proliferation. Oncoimmunology 2016;5:e1074375.

13. Hao S, He ZX, Yu KD, et al. New insights into the prognostic value of Ki-67 labeling index in patients with triple-negative breast cancer. Oncotarget 2016;7:24824-24831.

14. Regan MM, Francis PA, Pagani O, et al. Absolute benefit of adjuvant endocrine therapies for premenopausal women with hormone receptor-positive, human epidermal growth factor receptor 2-negative early breast cancer: TEXT and SOFT trials. J Clin Oncol 2016;34:2221-2231.

15. Shakeri H, Gharesouran J, Fakhrjou A, et al. DNA methylation assessment as a prognostic factor in invasive breast cancer using methylation-specific multiplex ligation dependent probe amplification. EXCLI J 2016;15:11.

16. Myers MB, Banda M, McKim KL, et al. Breast cancer heterogeneity examined by high-sensitivity quantification of PIK3CA, KRAS, HRAS, and BRAF mutations in normal breast and ductal carcinomas. Neoplasia 2016;18:253.

17. Kraege S, Stefan K, Juvale K, et al. The combination of quinazoline and chalcone moieties leads to novel potent heterodimeric modulators of breast cancer resistance protein (BCRP/ABCG2). Eur J Med Chem 2016;117:212.

18. Serpico V, Liepert AE, Boucher K, et al. The effect of previsit education in breast cancer patients: A study of a shared-decision-making tool. Am Surg 2016;82:259.

19. Du C, Yi X, Liu W, et al. MTDH mediates trastuzumab resistance in HER2 positive breast cancer by decreasing PTEN expression through an NFkappaB-dependent pathway. BMC Cancer 2014;14:869.

20. Ata A, Polat A, Serinsöz E, et al. Prognostic value of increased HER 2 expression in cancers of pancreas and biliary tree. Pathol Oncol Res 2015;21:831.

21. Murthy P, Kidwell KM, Schott AF, et al. Clinical predictors of long-term survival in HER2-positive metastatic breast cancer. Breast Cancer Res Treat 2016;155:589.

22. Liu L, Chen F, Zhao J, et al. Correlation between overall survival and other endpoints in metastatic breast cancer with second- or third-line chemotherapy: Literature-based analysis of 24 randomized trials. Bull Cancer 2016;103:336.

23. Shandiz FH, Shabahang H, Afzaljavan F, et al. Ki67 frequency in breast cancers without axillary lymph node involvement and its relation with disease-free survival. Asian Pac J Cancer Prev 2016;17:1347.

24. Kalinina EV, Berozov TT, Shtil AA, et al. Expression of genes of glutathione transferase isoforms GSTP1-1, GSTA4-4, and GSTK1-1 in tumor cells during the formation of drug resistance to cisplatin. Bull Exp Biol Med 2012;154:64.

25. Koleck TA, Bender CM, Sereika SM, et al. Polymorphisms in DNA repair and oxidative stress genes associated with pre-treatment cognitive function in breast cancer survivors: An exploratory study. Springerplus 2016;5:422. 